Commun. Korean Math. Soc. 28 (2013), No. 2, pp. 397-406

http://dx.doi.org/10.4134/CKMS.2013.28.2.397

\title{
AN IMPROVED BINOMIAL METHOD FOR PRICING ASIAN OPTIONS
}

\author{
Kyoung-Sook Moon And HongJoOng KIM
}

\begin{abstract}
We present an improved binomial method for pricing European- and American-type Asian options based on the arithmetic average of the prices of the underlying asset. At each node of the tree we propose a simple algorithm to choose the representative averages among all the effective averages. Then the backward valuation process and the interpolation are performed to compute the price of the option. The simulation results for European and American Asian options show that the proposed method gives much more accurate price than other recent lattice methods with less computational effort.
\end{abstract}

\section{Introduction}

An option is a financial instrument that gives its owner the right, but not the obligation, to buy or sell a pre-defined asset, called the underlying. By constructing a risk-neutral portfolio that replicates the return of holding an option, Black and Scholes [1] produce a closed-form solution for a European vanilla option's price. After derivation of the Black-Scholes equation, there have been traded many types of options including complex financial structures. Among them, an Asian option, which was introduced in Tokyo in 1987, is determined by the average asset price over the pre-defined time period. Because of the averaging feature, Asian options reduce the risk of market manipulation of the underlying asset at maturity and also the volatility inherent in the option. Therefore, Asian options are typically cheaper than European or American options. In practice, the average can be approximated by an arithmetic average or a geometric average. The geometric case has an analytic solution using the lognormal distribution of the underlying asset, see $[7,9]$. However, there is no

Received August 18, 2010.

2010 Mathematics Subject Classification. Primary 91B28, 65C20.

Key words and phrases. option pricing, binomial method, Asian option, American options.

This second author was supported by Basic Science Research Program through the National Research Foundation of Korea (NRF) funded by the Ministry of Education, Science and Technology (2011-000-5272). The first author was supported by the Gachon University Research Fund in 2012. 
closed-form solution for more popular arithmetic average-based Asian option, and therefore one needs to rely on numerical approximation. There have been studied various approaches. For analytic approximation, Levy [11], Vorst [15] and Ritchken et al. [13] calculate the probability density function of the arithmetic average case using the corresponding geometric average. Turnbull and Wakeman [14] improve the approximation using Edgeworth expansions. Later Curran [4] and Rogers and Shi [12] derive approximate formulae and bounds in the form of single and double integral. Alternatively one may use Monte Carlo simulation as in [6] or numerical methods for partial differential equation (see $[16])$.

However, all these methods cannot be simply applied to American-style Asian options. Therefore we focus on numerical schemes applicable for pricing American Asian option, especially binomial tree models. After the introduction by Cox et al. [3], the binomial method is a very popular method in computational finance due to its ease of implementation and simple application to American options. However Asian options based on the average of underlying asset prices present a difficult problem, since the number of average prices grows exponentially as the number of nodes of the tree increases. In order to solve this shortage of binomial method, Hull and white [8] compute a set of representative averages at each node. Their work makes the binomial model feasible for pricing Asian options but it generically suffers the lack of convergence (see $[2,5]$ ). Several modifications of the Hull and White model have been introduced since then. But some $[5,10,16]$ work only for specific cases or do not clearly specify the way to choose parameter values used in their methods. Or some [2] requires expensive computational costs such as large memory space or heavy computation.

This work presents a new binomial method to valuate Asian options efficiently and accurately. The proposed method can be applied to various European or American Asian options and numerical simulations in Section 5 show that the method computes accurately the price of the option in a simple way. The outline of the paper is as follows. Section 2 describes Asian option problem and Section 3 introduces some tree-based approaches for Asian option prices. Section 4 proposes a new binomial method for pricing Asian options and numerical experiments in Section 5 illustrates the efficiency and accuracy of the new method. A summary and some concluding remarks are given in Section 6 .

\section{Asian option problem}

Let us consider the price of the underlying asset as a stochastic process $\left\{S_{t}\right\}_{t \in[0, T]}$ on a suitable probability space $(\Omega, \mathcal{F}, \mathcal{P})$. Under risk neutral model, the evolution of the underlying is assumed to satisfy the stochastic differential equation:

$$
\mathrm{d} S(t)=r S \mathrm{~d} t+\sigma S \mathrm{~d} W(t), 0<t<T,
$$


where $r$ is a riskless interest rate, $\sigma$ is a volatility, $T$ is an expiration date, and $W(t)$ is a Brownian motion. Under the conventional assumptions of frictionless markets, the price of option can be computed by the conditional expectation of the discounted payoff,

$$
V(s, t)=e^{-r(T-t)} E[\Lambda(S(T), T) \mid S(t)=s],
$$

where $\Lambda(S(T), T)$ denotes the payoff function at expiration date $T$. For instance, the payoff of Asian call option (or average rate option) based on the arithmetic average of the underlying asset prices is

$$
\max \left(\frac{1}{T} \int_{0}^{T} S(\tau) d \tau-K, 0\right),
$$

where $K$ is a pre-defined exercise price. Here one obtains a discrete Asian option by replacing the continuous average $\frac{1}{T} \int_{0}^{T} S(\tau) d \tau$ by an arithmetic average $\frac{1}{N} \sum_{i=0}^{N} S\left(t_{i}\right)$ over $N+1$ time points $0=t_{0}<t_{1}<\cdots<t_{N}=T$.

The binomial method by Cox et al. [3] assumes that the asset price $S\left(t_{n}\right)$ at $t=t_{n}$ moves either up to $u S\left(t_{n}\right)$ for $u=\exp (\sigma \sqrt{\Delta t})>1$ or down to $d S\left(t_{n}\right)=$ $S\left(t_{n}\right) / u$ for $\Delta t=t_{n+1}-t_{n}$ for $n=0,1, \ldots, N-1$ with probabilities $p=$ $(\exp (r \Delta t)-d) /(u-d)$ or $1-p$, respectively. Then the standard binomial method calculates the payoffs of the option at expiry, $V\left(S\left(t_{N}\right), t_{N}\right)=\Lambda\left(S\left(t_{N}\right), t_{N}\right)$, and computes the option price $V(S(0), 0)$ by backward averaging,

(4) $V\left(S\left(t_{n}\right), t_{n}\right)=e^{-r \Delta t}\left(p V\left(S\left(t_{n+1}\right), t_{n+1}\right)+(1-p) V\left(S\left(t_{n+1}\right), t_{n+1}\right)\right)$,

where $n=N-1, N-2, \ldots, 0$. Since the number of averages prices grows exponentially as the number of time steps $N$ increases, the standard binomial method by Cox et al. [3] cannot be applied directly for pricing Asian options.

\section{Binomial methods for Asian options}

A forerunner of the binomial method for pricing Asian option is the one proposed by Hull and White [8], which considers a fixed strike European Asian call option based on the arithmetic average of the underling asset prices. The arithmetic average is calculated on $N+1$ asset prices during the option's life with the time step $\Delta t=T / N$ and $N$ is the number of time steps used to measure the price. Their model assumes that the price of the underlying evolves according to the CRR model by Cox et al. [3]. Let $(i, j)$ denote the node of the tree after $j$ upward movements and $i-j$ downward movements and $S(i, j)$ denote the price of the underlying asset at $(i, j)$ with $S(0,0)=S$. In a binomial method, the main obstacle for pricing an Asian option is the exponential increase of the number of possible payoffs. In order to solve this, Hull and White consider a finite set of representative averages at each node. The minimum and the maximum representative averages at time $i \Delta t, A_{\min }(i)$ and $A_{\max }(i)$ respectively, are of the form $S e^{ \pm m h}$, where $h$ is a fixed parameter 
and $m$ is the smallest integer such that

$$
\begin{aligned}
& A_{\min }(i)=S e^{-m h} \leq \frac{1}{i+1}\left(i A_{\min }(i-1)+d S(i-1,0)\right), \\
& A_{\max }(i)=S e^{m h} \geq \frac{1}{i+1}\left(i A_{\max }(i-1)+u S(i-1, i-1)\right) .
\end{aligned}
$$

Once $m$ is found, the other representative averages at each node $(i, \cdot)$ at time $i \Delta t$ are of the form $S e^{k h}$ where $k \in\{-m+1,-m+2, \ldots, m-1\}$. Let $A(i, j, k)$ be the $k^{\text {th }}$ representative average at the node $(i, j)$. Then the option price $C(i, j, k)$ corresponding to $A(i, j, k)$ is computed by the backward induction,

$$
C(i, j, k)=e^{-r \Delta t}\left(p C\left(i+1, j+1, k_{u}\right)+(1-p) C\left(i+1, j, k_{d}\right)\right),
$$

where $C\left(i+1, j+1, k_{u}\right)$ and $C\left(i+1, j, k_{d}\right)$ are option values corresponding to $((i+1) A(i, j, k)+u S(i, j)) /(i+2)$ and $((i+1) A(i, j, k)+d S(i, j)) /(i+2)$, respectively. $C\left(i+1, j+1, k_{u}\right)$ is computed by the linear interpolation between two option prices corresponding to the smallest representative average greater than $((i+1) A(i, j, k)+u S(i, j)) /(i+2)$ and the greatest representative average smaller than $((i+1) A(i, j, k)+d S(i, j)) /(i+2)$. A similar interpolation is applied to find $C\left(i+1, j, k_{d}\right)$. But, there are two weaknesses with this method. One is with the choice of the parameter $h$. The value of $h$ decides the number of representative values at each node and the numerical results by Hull and White show the declining pattern of the option price as $h$ decreases, which results from the linear interpolation that overestimates the option prices. According to Hull and White, such an overestimation effect should disappear asymptotically. Secondly, the numerical results show that for a given $h$ the option value is an increasing function of the number of time steps. That is, by increasing the number of time steps, the option value just increases (i.e., diverges). This lack of convergence is confirmed by Forsyth et al. [5] who showed that $h$ should be proportional to $\Delta t$ in order to obtain convergence. Hull and White, on the other hand, use fixed values of $h$, which leads to the increasing pattern of option values.

Forsyth et al. [5] improve the model of Hull and White by choosing $h$ as

$$
h=\frac{\alpha \sigma^{2} \Delta t}{2 \sqrt{T}},
$$

where $\alpha$ is a parameter. This $\alpha$ does a critical role in their model because it determines the number of representative averages to be found at each node. The results of Forsyth et al. present more stable convergence and accurate prices than those of Hull and White, but no theoretical rule is specified on how to choose the values of some parameters such as this critical parameter $\alpha$. In addition, the amount of computation of their model grows rapidly as $N$ increases because $h$ is proportional to $N$. In [2], Costabile et al. provide another efficient lattice model for pricing European and American Asian options based on the model of Hull and White. Their scheme can be applied to many 
European and American Asian options but the required computational cost is expensive as will be explained in detain in Section 4.

\section{Proposed binomial method}

Let us introduce a modified binomial method for pricing European- and American-type Asian options based on the arithmetic average of the underlying asset prices. When the standard lattice method by Cox et al. [3] is used for the Asian option, each path of the asset prices generates a different average price so that the number of whole average values increases exponentially in $N$, the number of time steps. Let $n_{i, j}$ denote the node of the tree after $i$ upward movements and $j$ downward movements. The set of the representative averages at $n_{i, j}$ is obtained by following steps. First, we compute the maximum and minimum average values from the initial node $n_{0,0}$ to $n_{i, j}, a_{i, j}^{\max }$ and $a_{i, j}^{\min }$, respectively. $a_{i, j}^{\max }$ is the average of the asset prices along the trajectory of $i$ initial upward movements followed by $j$ downward movements,

$$
a_{i, j}^{\max }=\frac{1}{i+j+1}\left(\frac{S\left(u^{i+1}-1\right)}{u-1}+\frac{S u^{i} d\left(1-d^{j}\right)}{1-d}\right)
$$

and $a_{i, j}^{\min }$ is the average along the trajectory of $j$ initial downward movements followed by $i$ upward movements,

$$
a_{i, j}^{\min }=\frac{1}{i+j+1}\left(\frac{S\left(1-d^{j+1}\right)}{1-d}+\frac{S u d^{j}\left(u^{i}-1\right)}{u-1}\right) .
$$

Then representative averages $a_{i, j}^{k}$ for the node $n_{i, j}$ are computed by

$$
a_{i, j}^{k}=a_{i, j}^{\min }+\frac{k}{i j}\left(a_{i, j}^{\max }-a_{i, j}^{\min }\right), \quad k=0,1, \ldots, i j .
$$

In [2], Costabile et al. estimated representative averages in a different way. They first considered the price path $\tau_{i, j}^{0}$, which results in $a_{i, j}^{\max }$ and set $a_{i, j}^{\max }$ to be the first representative average $A(i, j, 0)$. Then $k^{\text {th }}$ price path $\tau_{i, j}^{k}$ is obtained recursively by replacing the highest asset price, say $S^{\max }$, appearing in the previous path $\tau_{i, j}^{k-1}$ by $S^{\max } d^{2}, k=1, \ldots, i j$, and $k^{\text {th }}$ representative average $A(i, j, k)$ is obtained by the average asset price along $\tau_{i, j}^{k}$. Their algorithm needs to store all the path information and requires a procedure to search and replace the maximum asset price. Thus, the computational cost for their approach becomes expensive for a large lattice. The proposed method, on the other hand, uses the equation (5) to derive the representative averages, which does not require any memory storage for asset prices along each path and expedites the computation by avoiding search and replace step.

Once the representative averages at $T=N \Delta t$ are obtained, the payoff, for example, for the European Asian call option is optioned by

$$
\Lambda_{i, N-i}^{k}=\max \left\{a_{i, N-i}^{k}-K, 0\right\}
$$


for $k=0,1, \ldots, i(N-i), i=0,1, \ldots, N$ and is denoted by $c_{i, N-i}^{k}$ Then the backward induction procedure is performed,

$$
c_{i, j}^{k}=\exp ^{-r \Delta t}\left(p c_{i+1, j}^{k, \text { up }}+(1-p) c_{i, j+1}^{k, \text { down }}\right),
$$

where $c_{i+1, j}^{k, \text { up }}$ and $c_{i, j+1}^{k, \text { down }}$ are option prices corresponding to

$$
a_{i+1, j}^{k, \text { up }}=\frac{1}{i+j+2}\left((i+j+1) a_{i, j}^{k}+S u^{i+1} d^{j}\right)
$$

and

$$
a_{i, j+1}^{k, \text { down }}=\frac{1}{i+j+2}\left((i+j+1) a_{i, j}^{k}+S u^{i} d^{j+1}\right),
$$

respectively. The representative averages $a_{i+1, j}^{k}$ at node $n_{i+1, j}$ at time $(i+$ $j+1) \Delta t$ may not coincide with $a_{i+1, j}^{k, \text { up }}$. In order to overcome this problem, we compute $c_{i+1, j}^{k, \text { up }}$ by using the linear interpolation between two option prices corresponding to representative averages closest to $a_{i+1, j}^{k, \text { up }}$ as proposed in [8]. A similar procedure is applied to derive $c_{i, j+1}^{k, \text { down }}$.

When the early exercise is allowed as in, for example, an American Asian call option, the option price at the node $n_{i, j}$ is obtained by

$$
c_{i, j}^{k}=\max \left\{\exp ^{-r \Delta t}\left(p c_{i+1, j}^{k, \text { up }}+(1-p) c_{i, j+1}^{k, \text { down }}\right), a_{i, j}^{k}-K\right\} .
$$

A similar comparison is performed for the put option.

\section{Numerical results}

We present the computational results of the proposed scheme for Europeanand American-type Asian options based on the arithmetic average of the underlying asset price.

\subsection{European Asian options}

The simulation results of the proposed scheme for the European Asian call options with the initial asset price $S=100$, strike price $K=100$ and risk-free interest rate $r=0.1$ are compared with those from the schemes by Costabile et al. [2] and Forsyth et al. [5], where the prices of [5] are assumed to be (expected) exact option prices as in [2]. Two sets of parameters for the option are considered, one with a volatility of $\sigma=0.1$ and time to maturity $T=0.25$ (years) and the other with $\sigma=0.5$ and $T=5$. Table 1 compares the numerical results for various numbers of time steps, which shows the proposed scheme results in slightly faster convergence. 
TABLE 1. Option prices for European Asian options from the proposed method and the schemes by Costabile et al. and Forsyth et al.

\begin{tabular}{|c|c|c|c|c|}
\hline$N$ & $\begin{array}{r}\sigma=0.1 \\
\text { Costabile }\end{array}$ & $\begin{array}{l}T=0.25 \\
\text { Proposed }\end{array}$ & $\begin{array}{r}\sigma=0 . \\
\text { Costabile }\end{array}$ & $\begin{array}{l}T=5 \\
\text { Proposed }\end{array}$ \\
\hline 10 & 1.8381 & 1.8388 & 28.3491 & 28.4788 \\
\hline 20 & 1.8442 & 1.8451 & 28.2439 & 28.4161 \\
\hline 30 & 1.8466 & 1.8473 & 28.3478 & 28.4061 \\
\hline 40 & 1.8475 & 1.8483 & 28.3866 & 28.4052 \\
\hline 50 & 1.8485 & 1.8488 & 28.3899 & 28.4063 \\
\hline 60 & 1.8490 & 1.8492 & 28.3920 & 28.4074 \\
\hline 70 & 1.8492 & 1.8496 & 26.8899 & 28.4080 \\
\hline 80 & 1.8497 & 1.8498 & 28.3934 & 28.4098 \\
\hline 90 & 1.8499 & 1.8500 & 28.3875 & 28.4121 \\
\hline Forsyth & \multicolumn{2}{|c|}{1.8509} & \multicolumn{2}{|c|}{28.4003} \\
\hline
\end{tabular}

Figure 1 compares the schemes when the volatility of $\sigma=0.5$ and time to maturity $T=5$ (years). The figure also shows that the proposed scheme gives smoother and faster convergence than the method by Costabile et al.

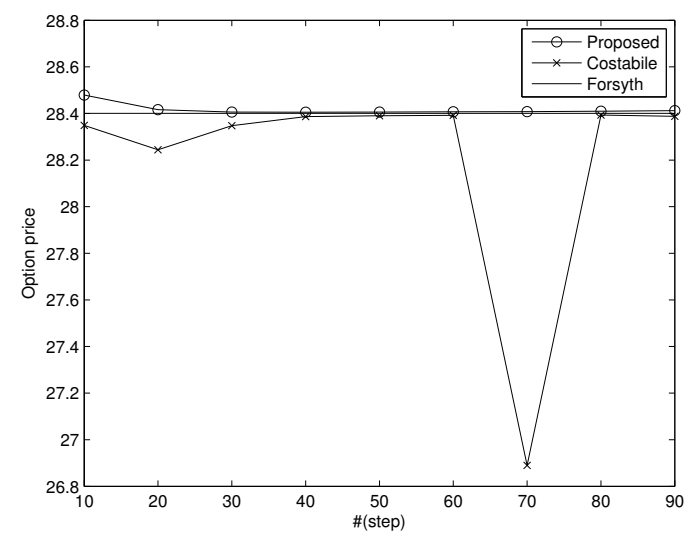

Figure 1. Option prices for European Asian options from the proposed method and the schemes by Costabile et al. and Forsyth et al. when $\sigma=0.5, T=5$.

\subsection{American Asian options}

For the American Asian options, the proposed scheme is compared with the schemes by Costabile et al. [2], Zvan et al. [16] and Klassen [10], where the prices of [16] and [10] are assumed to be (expected) exact option prices as in [2]. Zvan et al. used a numerical scheme based on the Crank-Nicolson method with flux limiter while Klassen implemented a binomial method with 512 time 
steps with Richardson extrapolation. Table 2 presents computational results for American Asian call options with $S=100, r=0.1, \sigma=0.4, T=1$ when the strike price $K$ and the number of time steps $N$ are varied. The model by Costabile et al. shows slower convergence for pricing American Asian options and thus requires a large number of time steps to achieve convergence, while the proposed method does not.

TABLE 2. Option prices for American Asian options with $S=$ $100, r=0.1, \sigma=0.4, T=1$ from the proposed method and the schemes by Costabile et al., Zvan et al. and Klassen.

\begin{tabular}{l|cc|cc|cc}
\hline & \multicolumn{2}{|c|}{$K=95$} & \multicolumn{2}{c|}{$K=100$} & \multicolumn{2}{c}{$K=105$} \\
$N$ & Costabile & Proposed & Costabile & Proposed & Costabile & Proposed \\
\hline 10 & 14.6269 & 14.6711 & 11.6977 & 11.7606 & 9.2388 & 9.2846 \\
20 & 14.6204 & 15.0967 & 11.5531 & 12.0311 & 9.0188 & 9.4988 \\
30 & 15.2235 & 15.2698 & 12.1183 & 12.1611 & 9.5461 & 9.5861 \\
40 & 15.2471 & 15.3639 & 12.1449 & 12.2316 & 9.5527 & 9.6363 \\
50 & 15.4162 & 15.4309 & 12.2652 & 12.2767 & 9.6597 & 9.6685 \\
60 & 15.4655 & 15.4781 & 12.3000 & 12.3093 & 9.6847 & 9.6914 \\
70 & 15.4976 & 15.5133 & 12.3206 & 12.3334 & 9.6979 & 9.7085 \\
80 & 15.5317 & 15.5405 & 12.3461 & 12.3523 & 9.7176 & 9.7220 \\
Zvan & \multicolumn{2}{|c|}{15.749} & \multicolumn{2}{c|}{12.497} & \multicolumn{2}{c}{9.825} \\
Klassen & 15.7747 & \multicolumn{3}{c}{9.8305} \\
\hline
\end{tabular}

Figure 2 compares the methods when $K=100$. As explained above, the proposed method results in smoother and faster convergence than that from Costabile et al.

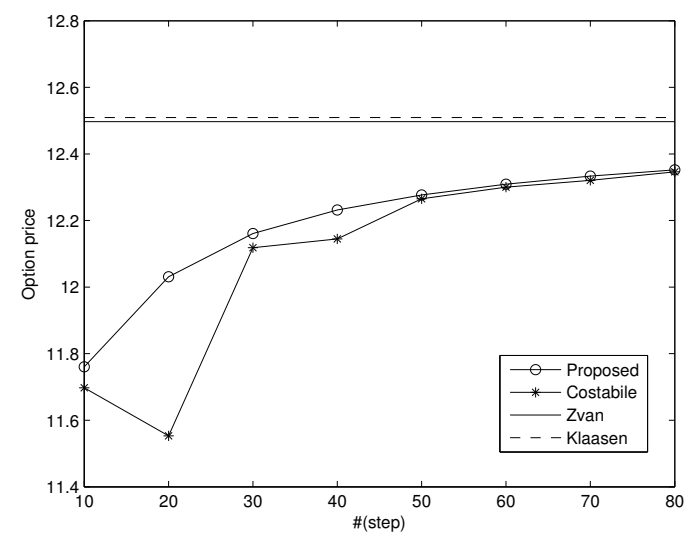

Figure 2. Option prices for American Asian options from the proposed method and the schemes by Costabile et al., Zvan et al. and Klassen when $S=100, r=0.1, \sigma=0.4, T=$ $1, K=100$. 
Table 3 shows the results of the American Asian options with $S=100, r=$ $0.1, \sigma=0.2, T=0.25$.

TABle 3. Option prices for American Asian options with $S=$ $100, r=0.1, \sigma=0.2, T=0.25$ from the proposed method and the schemes by Costabile et al., Zvan et al. and Klassen.

\begin{tabular}{l|cc|cc|cc}
\hline & \multicolumn{2}{|c|}{$K=95$} & \multicolumn{2}{c|}{$K=100$} & \multicolumn{2}{c}{$K=105$} \\
$N$ & Costabile & Proposed & Costabile & Proposed & Costabile & Proposed \\
\hline 10 & 6.9386 & 6.9401 & 3.0378 & 3.0421 & 0.9169 & 0.9211 \\
20 & 7.1302 & 7.1328 & 3.1035 & 3.1079 & 0.9478 & 0.9504 \\
30 & 7.2144 & 7.2168 & 3.1343 & 3.1372 & 0.9599 & 0.9616 \\
40 & 7.2626 & 7.2646 & 3.1510 & 3.1532 & 0.9664 & 0.9675 \\
50 & 7.2951 & 7.2967 & 3.1620 & 3.1638 & 0.9705 & 0.9712 \\
60 & 7.3180 & 7.3195 & 3.1697 & 3.1712 & 0.9732 & 0.9738 \\
70 & 7.3347 & 7.3361 & 3.1754 & 3.1766 & 0.9753 & 0.9757 \\
80 & 7.3484 & 7.3497 & 3.1799 & 3.1810 & 0.9768 & 0.9771 \\
Zvan & \multicolumn{2}{|c|}{7.521} & 3.224 & \multicolumn{2}{c}{1.009} \\
Klassen & 7.4660 & 3.2159 & \multicolumn{2}{c}{0.9882} \\
\hline
\end{tabular}

\section{Conclusions}

A binomial method for pricing Asian options is derived from the model of Hull and White. The algorithm of the proposed model is simple in the sense that it does not require large memory space nor heavy computation. The method can be applied to various types of European or American Asian options and all the parameter values can be easily derived from the Hull and White model. Algorithms to compute Greeks such as Delta or Gamma for Asian options will be derived in the future work.

\section{References}

[1] F. Black and M. Sholes, The pricing of options and corporate liabilities, The Journal of Political Economy 81 (1973), no. 3, 637-654.

[2] M. Costabile, I. Massabo, and E. Russo, An adjusted binomial model for pricing Asian options, Rev Quant Finan Acc 27 (2006), 285-296.

[3] J. Cox, S. Ross, and M. Rubinstein, Option pricing: A simplified approach, Journal of Financial Economics 7 (1979), 229-263.

[4] M. Curran, Beyond average intelligence, Risk 5 (1992), no. 10, 60 pages.

[5] P. A. Forsyth, K. R. Vetzal, and R. Zvan, Convergence of numerical methods for valuing path-dependent options using interpolation, Rev Derivatives Res 5 (2002), 273-314.

[6] P. Glasserman, Monte Carlo Methods in Financial Engineering, Springer-Verlag, New York, 2004.

[7] E. G. Haug, The Complete Guide to Option Pricing Formulas (2nd ed.), McGraw-Hill, 2007.

[8] J. Hull and A. White, Efficient procedures for valuing European and American pathdependent options, Journal of Derivatives 1 (1993), 21-31.

[9] A. Kemna and A. Vorst, A pricing method for options based on average asset values, Journal of Banking and Finance 14 (1990), 113-129. 
[10] T. R. Klassen, Simple, fast, and flexible pricing of Asian options, Journal of Computational Finance 4 (2001), 89-124.

[11] E. Levy, Pricing European average rate currency options, Journal of International Money and Finance 11 (1992), 474-491.

[12] L. C. G. Rogers and Z. Shi, The value of an Asian option, Journal of Applied Probability 32 (1995), 1077-1088

[13] L. Sankarasubramanian, P. Ritchken, and A. M. Vijh, The valuation of path dependent contracts on the average, Management Science 39 (1991), 1202-1213.

[14] S. M. Turnbull and L. M. Wakeman, A quick algorithm for pricing European average options, Journal of Financial and Quantitative Analysis 26 (1991), 377-389.

[15] T. Vorst, Prices and hedge ratios of average exchange rate options, International Review of Financial Analysis 1 (1992), 179-193

[16] R. Zvan, P. A. Forsyth, and K. R. Vetzal, Robust numerical methods for pde models of Asian options, Journal of Computational Finance 1 (1998), 39-78.

KYoung-SOOK MoOn

Department of Mathematics \& Information

GaChon University

GyeONGgi 461-701, KoreA

E-mail address: ksmoon@gachon.ac.kr

HongJoOng Kim

Department of Mathematics

Korea UNIVERSITY

SEOUl 136-701, Korea

E-mail address: hongjoong@korea.ac.kr 